\title{
Urinary lodine Kinetics after Oral Loading of Potassium lodine
}

\author{
NOBORU TAKAMURA, AIKo HAMADA*, NINPEI YAMAGUCHI, NAHOE MATSUSHITA**, \\ IGOR TARASIUK***, TOSHINORI OHASHI ${ }^{*}$, KIYOSHI AOYAGI, MARIKO MINE* \\ AND SHUNICHI YAMASHITA* \\ Department of Public Health, Nagasaki University Graduate School of Biomedical Sciences, Nagasaki 852-8523, Japan \\ *Atomic Bomb Disease Institute, Nagasaki University Graduate School of Biomedical Sciences, Nagasaki 852-8523, Japan \\ **Nutrition Management Room, Nagasaki University Hospital, Nagasaki 852-8523, Japan \\ ***Gomel State Medical Institute, Gomel, The Republic of Belarus \\ \#Pharmaceutical Research Laboratory, Hitachi Chemical Co., Ltd., Ibaragi 317-8555, Japan
}

\begin{abstract}
We studied the kinetics of iodine in various forms, in order to establish appropriate guidelines of iodine prophylaxis for thyroid blockade at nuclear emergency in "iodine rich areas", such as Japan. First, the effect of equivalent dose of potassium iodide (KI) (solution vs. tablet) was evaluated with excretions of urinary iodine (UI) at Nagasaki, Japan, and it was revealed that there was no difference of iodine kinetics between solution and tablet. We also performed the same study at Gomel, Belarus, which was known to be an iodine deficient area, and obtained the same results. Second, the kinetics of iodine included in "iodine rich" food was also evaluated with excretions of UI. Interestingly, the ratio of UI excretion with iodine rich food was significantly lower than that with KI tablets until $6 \mathrm{~h}$ after the intake (ANOVA, $\mathrm{p}=0.02$ ). These results proved that 1 ) KI solution as well as its tablet is useful for prophylaxis and 2) prophylaxis by iodine rich food is not effective for rapid blockade of thyroid gland at nuclear emergency. Finally, we emphasized that the approach from social medicine is definitely important to establish an effective iodine prophylaxis.
\end{abstract}

Key words: Potassium iodide, Iodine-rich area, Urinary iodine, Thyroid, Radiation emergency

(Endocrine Journal 50: 589-593, 2003)

IT is well known that after the Chernobyl nuclear power accident plant, there was a marked increase in thyroid cancer among young children $[1,2]$. This strongly suggests a causal relationship between internal irradiation of the iodine isotope, possibly $\mathrm{I}^{131}$, and the occurrence of thyroid cancer. Although potassium iodide (KI) was available in the former USSR, because of poor communication and general secrecy about the accident, the effective distribution of KI was not conducted [3]. This indicates the importance of thyroid blockade by stable iodine prophylaxis just after a nuclear accident [4]. Especially in iodinedeficient areas, such preparedness is very important

Received: March 10, 2003

Accepted: June 12, 2003

Correspondence to: Noboru TAKAMURA, M.D., Ph.D., Department of Public Health, Nagasaki University Graduate School of Biomedical Sciences, 1-12-4 Sakamoto, Nagasaki 852-8523 Japan to protect the thyroid gland from iodine isotopes, in order not to repeat the tragedy of Chernobyl.

In contrast, Japan is one of the most "iodine-rich" areas in the world, because of the large amount of iodine contained in Japanese foods, such as seaweed and fish. According to our recent study, the median level of urinary iodine in Japan was $362.6 \mu \mathrm{g} / \mathrm{L}$ among schoolchildren and $612.0 \mu \mathrm{g} / \mathrm{L}$ among adults [5]. In this country, a guideline for iodine prophylaxis has not been established, since the necessity of such action in iodine-rich areas is controversial. There has been no study to evaluate the kinetics of iodine contained in foods, which is essential to solve this problem.

Since the accident of the uranium processing facility at the Tokaimura Nuclear Complex in 1999 [6], the discussion for effective prevention against unexpected nuclear accidents has taken place in Japan, and the need of iodine prophylaxis, especially for young children, based on the results of epidemiological study 
of Chernobyl was emphasized [7].

In 1977, International Atomic Energy Agency (IAEA) established the interventional criterion for a radiation emergency as $100 \mathrm{mSv}$ [8]. Furthermore, in 1989, a World Health Organization (WHO) committee reviewed the issue of $\mathrm{KI}$ use and outlined that $\mathrm{KI}$ should be administered to all population groups in the immediate vicinity of a reactor if a projected dose exceeded the national intervention level, and that KI should also be given to pregnant and nursing women and children under 16, even at sites far from the reactor [9]. Generally, stable iodine for a radiation emergency is to be provided in tablet form. But for children, who are the most sensitive to irradiation, tablets are sometimes difficult to swallow.

In order to address these issues, we compared the kinetics of equivalent dose of KI in solution and tablet form with urinary iodine (UI) excretion. We then evaluated the kinetics of iodine included in "iodine-rich" food with UI levels. The results obtained from this study will help to establish guidelines for iodine prophylaxis in iodine-rich countries.

\section{Materials and Methods}

\section{Study participants}

Based on epidemiological study results of Chermobyl [7], studies were carried out in healthy, euthyroid male volunteers, ages 18 to 24, in Nagasaki, Japan and Gomel, Republic of Belarus. Ethical approval was obtained from the special committee of the Nagasaki University School of Medicine (project registration no. 14032639). Permission was also obtained from the ethical committee of the Gomel Medical Institute. Before the study, informed consent was obtained from each participant. None of the individuals had any history of chronic disease, thyroid disease, or other systemic disorders. During trials, no complications, such as iodine induced thyrotoxicosis and hypersensitivity reactions were observed.

\section{Administration of tablet or solution of KI}

For the first trial, four (Nagasaki) and five (Gomel) participants were given $2 \mathrm{KI}$ tablets (total $100 \mathrm{mg}$ $\mathrm{KI}$ including $76 \mathrm{mg}$ iodine). Blood samples were collected from each participant before treatment, and spot urine samples were collected before treatment and at 1, 2, 6, 12 and $24 \mathrm{~h}$ after treatment. One week after the first trial, the same subjects were given 100 $\mathrm{mg} / 10 \mathrm{ml}$ of KI solution (including $76 \mathrm{mg}$ iodine), dissolved in deionized water. Blood samples were collected from each participant before treatment, and spot urine samples were collected before treatment and at 1, 2, 6, 12 and $24 \mathrm{~h}$ after treatment.

Another four (Nagasaki) and four (Gomel) participants were given $100 \mathrm{mg} / 10 \mathrm{ml}$ of KI solution for the first trial, and were given 2 tablets (total $100 \mathrm{mg}$ including $76 \mathrm{mg}$ iodine) of $\mathrm{KI}$ for the second trial. Blood and urine samples were collected in the same manner. The UI concentrations obtained were adjusted by the urinary creatinine concentrations of the same samples.

\section{Evaluation of iodine kinetics after consumption of} iodine rich food

Iodine rich food, containing considerable amounts of seaweed, was prepared according to the chart of Japanese dietary reference intakes. We prepared three types of food, i.e. high iodine food, middle iodine food, and low iodine food (Fig. 1). High iodine food was prepared with $40 \mathrm{~g}$ of "dry sea tangle," and middle

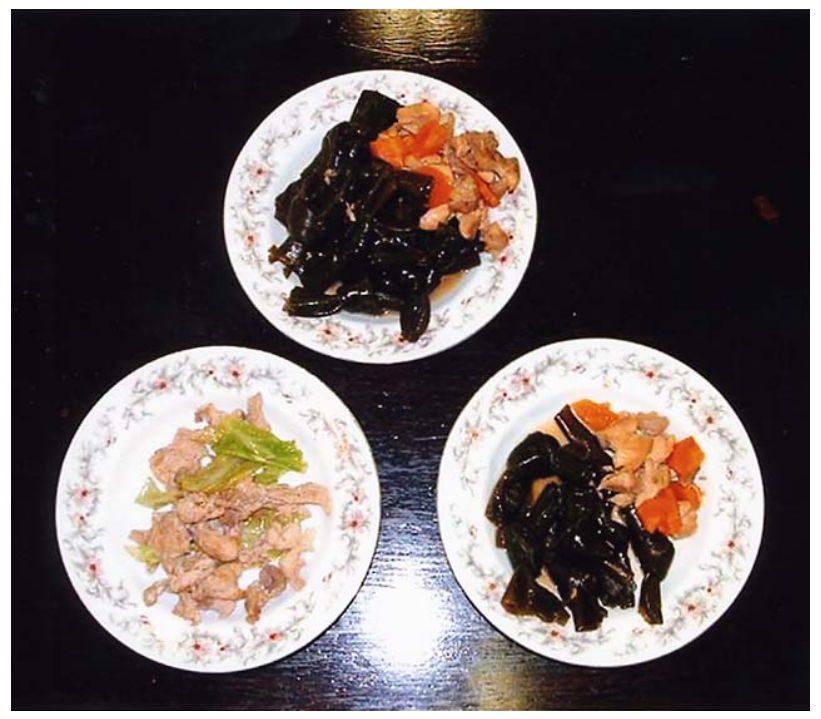

Fig. 1. High iodine food (upper), middle iodine food (lower right), and low iodine food (lower left). High iodine food includes $40 \mathrm{~g}$ of "dry sea tangle", chicken and carrots. Middle iodine food was prepared with half the amount of high iodine food. Low iodine food includes pork and cabbage. 
iodine food was prepared with half of that amount. For low iodine food, we used "baked pork." Each food was prepared for five participants.

Blood samples were collected from each participant before eating, and urine samples were also collected during $0-2,2-6,6-12$ and $12-24 \mathrm{~h}$ after meals. After the calculation of urinary volumes, spot urine samples were collected from accumulated urines for the following study. Recovery ratio of iodine was calculated as (cumulative excretion amount of iodine $(\mathrm{mg}) /$ total iodine $(\mathrm{mg})) \times 100(\%)$.

For controls, three participants were given two tablets $(100 \mathrm{mg}, 76 \mathrm{mg}$ total iodine dose) and one tablet of KI, and urine samples were collected and evaluated by the same protocol.

\section{Measurement of UI}

The UI concentrations were measured by "simple microplate method," based on the Sandel-Kolthoff reaction [10], which incorporates both the reaction and the digestion process into a microplate format. Details of this method are described elsewhere [11]. In brief, using a specially designed sealing cassette to prevent loss of vapor and cross-contamination among plates, ammonium persulfate digestion was performed in a polypropylene Serocluster 96-wellplate in an oven at $110^{\circ} \mathrm{C}$ for $60 \mathrm{~min}$. After the digestion, mixture was transferred to a transparent microplate and the Sandell-Kolthoff reaction was performed at $25^{\circ} \mathrm{C}$ for $30 \mathrm{~min}$, urinary iodine was measured by a microplate reader at $405 \mathrm{~nm}$. The sensitivity of this method was $>10 \mu \mathrm{g} / \mathrm{L}$.

For the measurement of iodine concentrations in foods, each food, containing a volume identical to that which participants received, was completely reduced to paste form. The paste was heated to $90^{\circ} \mathrm{C}$ for $1 \mathrm{~h}$ and then centrifuged. The supernatant obtained was used to measure iodine using the simple microplate method, and total iodine volume was calculated.

\section{Statistical analysis}

Data were analyzed using the Statistical Analysis System, version 6.12 (SAS Institute, Inc. Cary, NC). The Mann-Whitney test was used to compare the means of UI concentrations after intake of tablet and solution of KI. Analysis of variance was used to test differences in means between iodine-rich food and KI solution. A p value less than 0.05 was regarded as significant.

\section{Results}

Comparison of UI concentrations after intake of tablet and solution of $K I$

In Nagasaki, the average UI concentration before the experiment was $0.25 \mathrm{mg} / \mathrm{gCr}(406 \mu \mathrm{g} / \mathrm{L})$. There was no difference of kinetics between KI tablet and solution (Fig. 2). Until $2 \mathrm{~h}$ after intake (both solution and tablet), a remarkable excess of UI excretion was observed. After that, UI excretion decreased significantly. Also in Gomel, there was no difference in kinetics between solution and tablet $(p>0.05)$. The average UI concentration before the experiment was
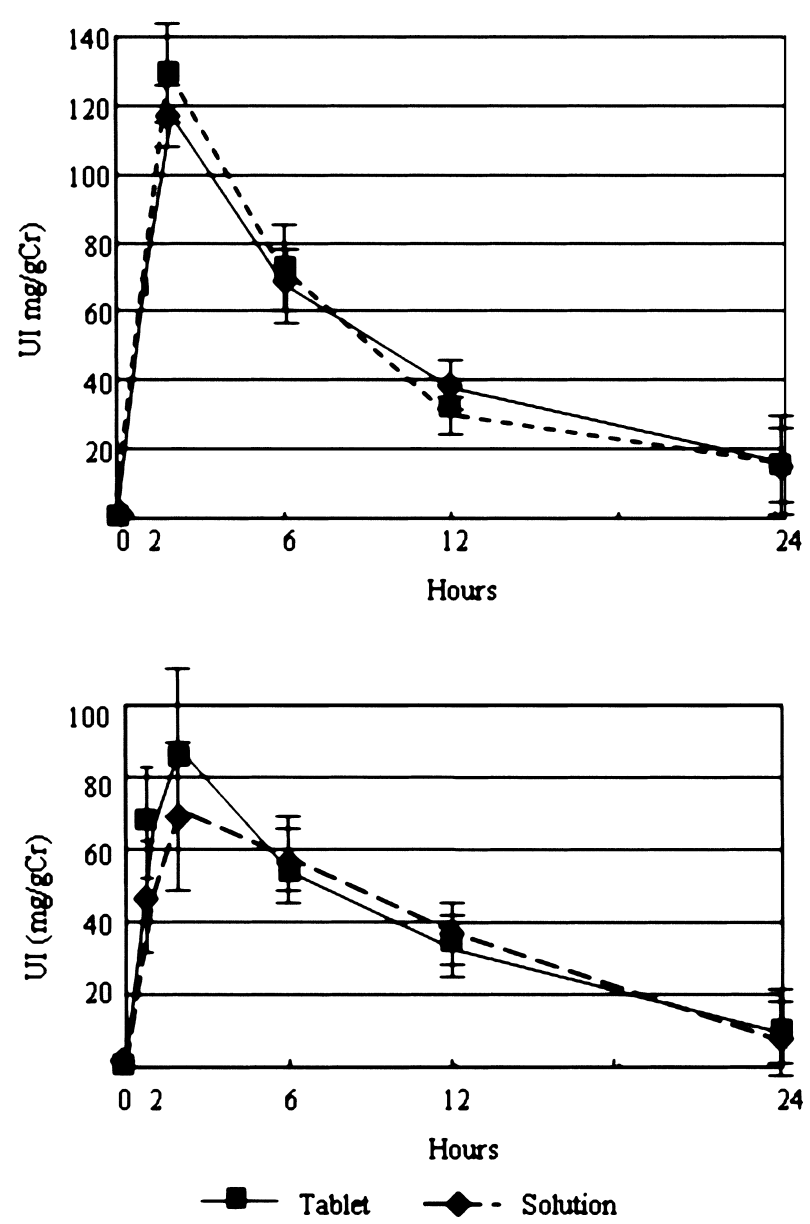

Fig. 2. UI concentrations after intake of tablet and solution of $\mathrm{KI}$ in Nagasaki (upper) and in Gomel (lower). 


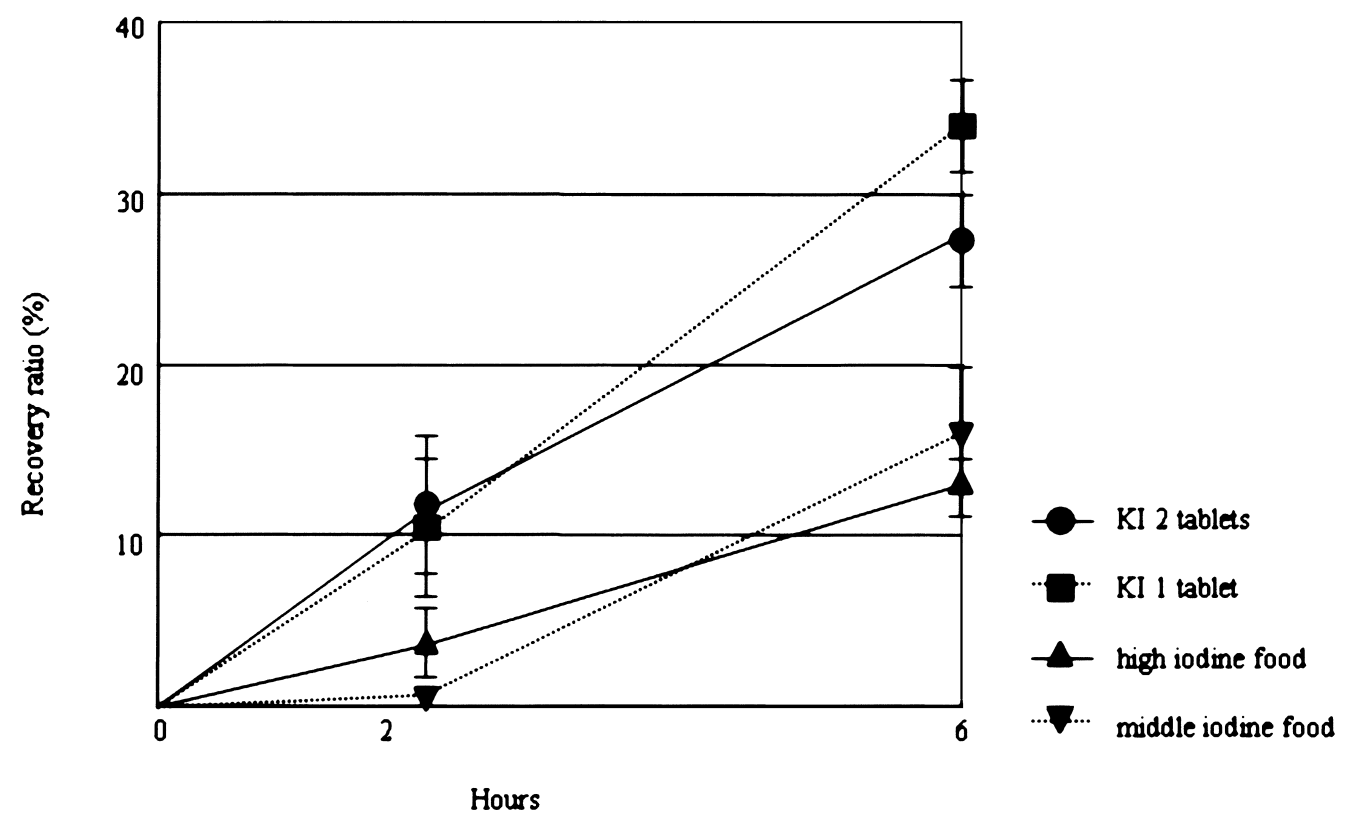

Fig. 3. Recovery ratio of iodine excretion with iodine rich foods (high and middle).

\section{$0.12 \mathrm{mg} / \mathrm{gCr}(143.3 \mu \mathrm{g} / \mathrm{L})$.}

\section{Evaluation of iodine kinetics after consumption of} iodine-rich food

Mean amounts of iodine among high iodine food, middle iodine food, and low iodine food were $16.7 \mathrm{mg}, 9.6 \mathrm{mg}$ and $0 \mathrm{mg}$, respectively. As shown in Fig. 3, the ratio of iodine excretion with iodine-rich food was significantly lower than that of KI tablets (including $76 \mathrm{mg}$ and $38 \mathrm{mg}$ iodine) until $6 \mathrm{~h}$ after the intake $(\mathrm{p}=0.02)$.

\section{Discussion}

In this study, we have clearly shown the following two facts. First, there was no difference in the kinetics of solution and tablet KI between iodine rich and iodine deficient areas. Second, the ratio of iodine excretion of iodine-rich foods was much slower than that of KI. These results suggest that iodine prophylaxis is possible by KI solution, as well as KI tablets, but is not possible by iodine-rich foods, even in iodinerich countries.

For effective iodine prophylaxis during a radiation emergency, the most important age group to target is young children. Children up to six years of age should be strictly protected from the radiation fallout, according to experience of the accident at the Chernobyl nuclear power plant [7]. In almost all established guidelines for iodine prophylaxis, regardless of country, it is indicated that KI tablets should be given immediately after the radiation emergency, even to young children. In practice, it is very difficult for such children to easily take tablets even with water. Now under the supervision of the Japanese Government, a special commission on the iodine prophylaxis is preparing a protocol for radiation emergency in Japan. The main topic of this commission is the effectiveness of prophylaxis for young children. An idea proposed by this commission is that children under age seven should intake KI solution, instead of tablets. In detail, the proposal is that a solution containing $12.5 \mathrm{mg}, 25 \mathrm{mg}$ or $38 \mathrm{mg}$ iodide (as a form of $\mathrm{KI}$ ) should be given to neonates, one month to three yearold, and three year to six year-old, respectively. Before finalizing this protocol, it is necessary to study the kinetics of KI solutions and tablets to confirm that they are comparable.

Our study shows that there is no difference in the kinetics of either form in iodine-rich (Nagasaki) and iodine-deficient area (Gomel). It showed that KI solution, as well as tablet, can block the thyroid after a radiation emergency in iodine-rich areas, as well as in deficient areas. 
In iodine-rich areas, the necessity of thyroid blockade by stable iodine prophylaxis has been discussed. One opinion is that thyroid blockade is not necessary since in such areas people usually eat iodine-rich foods, such as seaweed and fish. It is estimated that the intake of iodine in Japan is between 200 to $30,000 \mu \mathrm{g} / \mathrm{day}$, but there is no report on the kinetics of iodine in foods.

Our results showed that thyroid blockade by iodinerich food is not sufficient or adequate in case of a radiation emergency. Mean amounts of iodine among high and middle iodine food were $16.7 \mathrm{mg}$ and $9.6 \mathrm{mg}$, respectively. Our study showed that the average time for the intake of iodine-rich food (equivalent to less than a half dose of one KI tablet) was over $1 \mathrm{~h}$ (data not shown). The recommended KI dosage regimens were $100 \mathrm{mg}$ for adults in most countries. This suggests that the thyroid blockade by iodine-rich food is not realistic even in iodine-rich areas. Furthermore, our study showed that the ratio of iodine excretion with iodine-rich foods was significantly lower than that with KI tablets until $6 \mathrm{~h}$ after the intake. For effective blockade, rapid uptake of iodine into thyroid is very important. It was reported that the effect of blockade would significantly decrease after $6 \mathrm{~h}$ of the inhalation of $\mathrm{I}^{131}[12,13]$. With this in mind, blockade with iodine-rich food is not an appropriate method, even in iodine-rich areas.

Our present study showed that i) KI solution as well as its tablet is useful for prophylaxis and ii) prophylaxis by iodine-rich food is not effective for rapid blockade of the thyroid gland during a nuclear emergency. In conclusion, KI solution will be useful for smooth implementation of prophylaxis, especially for children, and furthermore, the development of drug delivery systems will enable smooth and systematic iodine prophylaxis at the time of a radiation emergency.

\section{Acknowledgement}

This study was financially supported by The Nuclear Safety Research Association, Japan.

\section{References}

1. Souchkevitch GN, Tsyb AF (1997) Health Consequences of the Chernobyl Accident: results of the IPEHCA pilot projects and related national programmes. WHO, Geneva, 519.

2. Yamashita S, Shibata Y (1997) Chernobyl A Decade. ICS, 1156, Excerpta Medica, Amsterdam, 613.

3. Mettler FA, Royal HD, Hurley JR (1992) Administration of stable iodine to the population around the Chernobyl nuclear power plant. J Radiol Prot 12: 159165.

4. Becker DV, Zanzonico P (1997) Potassium iodide for thyroid blockade in a reactor accident: administrative politics that govern its use. Thyroid 7: 193-197.

5. Ishigaki K, Namba H, Takamura N, Saiwai H, Parshin V, Ohashi T, Kanematsu T, Yamashita S (2001) Urinary iodine levels and thyroid disease in children; Comparison between Nagasaki and Chernobyl. Endocr $J$ 48: 591-595.

6. IAEA Issues Report on the preliminary fact-finding mission following the accident at the nuclear fuel processing facility in Tokaimura, Japan (1999) International Atomic Energy, Vienna, 1-44.
7. Shibata Y, Yamashita S, Masyakin VB, Panasyuk GD, Nagataki S (2001) 15 years after Chernobyl: new evidence of thyroid cancer. Lancet 358: 1965-1966.

8. Intervention criteria in a nuclear or radiation emergency. IAEA SS109 (1994) International Atomic Energy, Vienna.

9. Guidelines for iodine prophylaxis following nuclear accidents. Environmental Health Series (1989) WHO, Copenhagen.

10. Sandel EB, Kolthoff IM (1937) Simple microplate method for determination of urinary iodine. Clin Chem 46: 529-536.

11. Ohashi T, Yamaki M, Pandav CS, Karmarkar MG, Irie M (2000) Simple microplate method for determination of urinary iodine. Clin Chem 46: 529-536.

12. NCRP. Report No. 55 (1977) Protection of the thyroid gland in the event and potential pregnant women.

13. Nagataki S (1996) Other factors regulating thyroid function. In: Braverman LE and Utiger RD (eds) Werner \& Ingbar's The Thyroid. A fundamental Clinical Text. 7th ed. Lippincott-Raven Publishers, Philadelphia, 241-246. 